\title{
ERRATUM: Prognostic Implications of Extranodal Extension in Relation to Colorectal Cancer Location
}

\section{Chan Wook Kim ${ }^{1}$, Jihun Kim², Yangsoon Park', Dong-Hyung Cho ${ }^{3}$, Jong Lyul Lee', Yong Sik Yoon ${ }^{1}$, In Ja Park', Seok-Byung Lim Chang Sik Yu', Jin Cheon Kim}

Departments of ${ }^{1}$ Surgery and ${ }^{2}$ Pathology, Institute of Innovative Cancer Research, Asan Medical Center, University of Ulsan College of Medicine, Seoul, ${ }^{3}$ School of Life Science, Kyungpook National University, Daegu, Korea

While revising our manuscript according to the reviewers' comments, we changed the data of five cells in Table 3 . Among these, three changes were made correctly, while two were by our careless mistake. The correct numbers should have been 14 $(8.7 \%)$ instead of $14(5.4 \%)$ in the cell of "multiple route" and "ENE(+)", and 0.031 instead of 0.168 in the of "multiple route" and "p-value", respectively. Based on this, one sentence at the end of "Results" section (5. Recurrence patterns according to tumor location and ENE status) needs to be rephrased as below.

Original: "In rectal cancer patients, however, LN and multiple route recurrences (e.g., hematogenous and LN, or hematogenous and peritoneal seeding) were significantly more frequent in ENE-positive than in ENE-negative patients (Table 3)."

Corrected: "In rectal cancer patients, however, multiple route recurrence (e.g., hematogenous and LN, or hematogenous and peritoneal seeding) was significantly more frequent in ENE-positive than in ENE-negative patients (Table 3)."

We deeply apologize for our careless mistakes that have caused confusion to the readers of this article. Furthermore, we sincerely appreciate Professor Jeonghyun Kang (Department of Surgery, Gangnam Severance Hospital, Yonsei University College of Medicine), who pointed out our mistakes to the editors of Cancer Research and Treatment.

*DOI of original article: https:// doi.org/10.4143/ crt.2018.392 